\title{
The Effect of Early Visual Deprivation on the Neural Bases of Auditory Processing
}

\author{
Maria J. S. Guerreiro, Lisa Putzar, and Brigitte Röder \\ Brain Psychology and Neuropsychology, Institute for Psychology, University of Hamburg, D-20146 Hamburg, Germany
}

Transient congenital visual deprivation affects visual and multisensory processing. In contrast, the extent to which it affects auditory processing has not been investigated systematically. Research in permanently blind individuals has revealed brain reorganization during auditory processing, involving both intramodal and crossmodal plasticity. The present study investigated the effect of transient congenital visual deprivation on the neural bases of auditory processing in humans. Cataract-reversal individuals and normally sighted controls performed a speech-in-noise task while undergoing functional magnetic resonance imaging. Although there were no behavioral group differences, groups differed in auditory cortical responses: in the normally sighted group, auditory cortex activation increased with increasing noise level, whereas in the cataract-reversal group, no activation difference was observed across noise levels. An auditory activation of visual cortex was not observed at the group level in cataract-reversal individuals. The present data suggest prevailing auditory processing advantages after transient congenital visual deprivation, even many years after sight restoration.

Key words: auditory processing; fMRI; sight restoration; speech processing; visual deprivation

\section{Significance Statement}

The present study demonstrates that people whose sight was restored after a transient period of congenital blindness show more efficient cortical processing of auditory stimuli (here speech), similarly to what has been observed in congenitally permanently blind individuals. These results underscore the importance of early sensory experience in permanently shaping brain function.

\section{Introduction}

Individuals born blind as a result of dense bilateral cataracts and who later regain sight after cataract removal provide a unique model to investigate the effect of early sensory experience in shaping brain and behavior in humans. This research has demonstrated severe impairments in visual and multisensory processing, despite many years of visual recovery (for review, see Lewis and Maurer, 2005; Lewkowicz and Röder, 2012). In contrast, the extent to which transient congenital visual deprivation affects auditory processing has been investigated considerably less. Nevertheless, the few studies including unimodal auditory conditions in their designs suggest that auditory processing does not differ

\footnotetext{
Received July 2, 2015; revised Nov. 20, 2015; accepted Dec. 21, 2015.

Author contributions: L.P. and B.R. designed research; L.P. performed research; M.J.S.G. and L.P. analyzed data; M.J.S.G. and B.R. wrote the paper.

This research was supported by European Research Council Grant ERC-2009-AdG249425 (CriticalBrainChanges; B.R.). We thank Ines Goerendt for her help in assisting with data collection and Björn Zierul for his assistance in running some of the imaging preprocessing steps.

The authors declare no competing financial interests.

Correspondence should be addressed to Maria J. S. Guerreiro, Biological Psychology and Neuropsychology, Institute for Psychology, University of Hamburg, Von-Melle-Park 11, D-20146 Hamburg, Germany. E-mail: maria.guerreiro@uni-hamburg.de.

DOI:10.1523/JNEUROSCI.2559-15.2016

Copyright $\odot 2016$ the authors $\quad 0270-6474 / 16 / 361620-11 \$ 15.00 / 0$
}

between cataract-reversal individuals and normally sighted controls (Putzar et al., 2007, 2010, 2012).

Conversely, research in permanently blind individuals has documented extensive evidence for behavioral compensation and brain reorganization during auditory processing, particularly in congenitally blind individuals. In fact, blind individuals display higher auditory processing skills in a number of tasks, including voice processing (Föcker et al., 2012) and speech discrimination (Niemeyer and Starlinger, 1981; Dietrich et al., 2013). These enhanced auditory abilities have been linked to neuroplasticity within auditory brain areas (i.e., intramodal plasticity; Röder et al., 1996; Elbert et al., 2002; Gougoux et al., 2009), as well as within brain areas that are typically dedicated to vision in sighted controls (i.e., crossmodal plasticity). Indeed, a number of studies have shown that blind individuals, unlike sighted controls, activate "visual" brain areas - in particular, the calcarine sulcus and fusiform gyrus - during speech and voice processing (Röder et al., 2002; Hertrich et al., 2009; Bedny et al., 2011, 2012; Dietrich et al., 2013; Hölig et al., 2014a,b).

In light of the extensive evidence for brain reorganization during auditory processing in permanently blind individuals, two studies have investigated whether such reorganization remains after vision is restored. In one of these studies (Saenz et al., 2008), two sight-recovery individuals were shown to exhibit both visual 
and auditory motion-selective responses within the typically visual motion-selective middle temporal area MT, suggesting that crossmodal responses coexist with regained visual responses. In a longitudinal study (Dormal et al., 2015), another sight-recovery individual was shown to exhibit occipital activations in response to a range of auditory stimuli both before and after surgery, which nevertheless decreased over time. These studies suggest that, despite the lack of behavioral differences in auditory tasks between cataract-reversal individuals and normally sighted controls (Putzar et al., 2007, 2010, 2012), there might be differences in the neural bases of auditory processing. Importantly, the participants in the studies above had been visually deprived for an extensive period of time (i.e., 35-43 years), which in two (of the three) cases started only at the age of 3 years. As such, the role of visual experience within the first months of life (or lack thereof) on the development of the neural bases of auditory processing remains to be investigated.

The goal of the present study was to investigate the effect of transient congenital visual deprivation on the neural bases of auditory processing. To this end, we used a speech-in-noise task in combination with functional magnetic resonance imaging (fMRI) to evaluate the presence of intramodal plasticity and crossmodal plasticity in cataract-reversal individuals. Research in normally sighted individuals has shown that increasing the energetic masking of speech stimuli, by adding multi-talker babble, results in an increase of posterior superior temporal gyrus activation (Wong et al., 2008, 2009). In permanently blind individuals, including children, speech perception has been shown to elicit cortical responses in "visual" brain areas, in particular the calcarine sulcus and fusiform gyrus (Röder et al., 2002; Hertrich et al., 2009; Bedny et al., 2011, 2012, 2015; Dietrich et al., 2013; Hölig et al., 2014a,b). Intramodal plasticity, if present, should be observed in the present study as a difference between groups in auditory cortex activation, whereas crossmodal plasticity should be observed as a difference between groups in visual cortex activation in response to auditory stimulation.

\section{Materials and Methods}

\section{Participants}

A group of nine individuals with a history of dense bilateral congenital cataracts (aged 18-51 years; mean $\pm \mathrm{SD}, 35.67 \pm 12.09$; seven females), whose cataract-removal surgery took place between the ages of 3 and 24 months (mean $\pm \mathrm{SD}, 12.67 \pm 8.37$ ), and a group of nine individuals matched for age and education level (aged 19-56 years; mean \pm SD, $34.89 \pm 10.71$; seven females) participated in this study. Clinical and demographic information are presented in Table 1.

All participants were right-handed, native German speakers and reported normal hearing. In the group of cataract-reversal individuals, visual acuity ranged from $20 / 50$ to $20 / 200$, with a mean of 20/70. Normally sighted controls had normal or corrected-to-normal visual acuity. During testing, the cataract-reversal participants were fitted with individually tailored optical corrections that focused the eyes on the testing distance.

The study was approved by a local ethics committee (Ärztekammer Hamburg, protocol 2502), and, in accordance with the Declaration of Helsinki, written informed consent was obtained from each participant before testing.

\section{Experimental design}

The experiment reported here was part of a larger study comprising visual, auditory, and audiovisual stimulation conditions and aimed at investigating multisensory processing in cataract-reversal individuals (parts of this study have been published previously; see Putzar et al., 2007, 2010; Guerreiro et al., 2015). In the present study, we were interested in assessing the effect of early visual deprivation on the neural bases of auditory processing. To this end, we compared cortical responses of
Table 1. Clinical and demographic information

\begin{tabular}{|c|c|c|c|c|c|}
\hline Participant & Sex & $\begin{array}{l}\text { Age } \\
\text { (years) }\end{array}$ & $\begin{array}{l}\text { Visual } \\
\text { deprivation } \\
\text { (months) }\end{array}$ & $\begin{array}{l}\text { Visual acuity } \\
\text { in the better } \\
\text { eye (decimal) }\end{array}$ & Additional details \\
\hline $\mathrm{C} 1$ & $\mathrm{~F}$ & 29 & 6 & 0.20 & $\begin{array}{l}\text { Manifest nystagmus, microcornea, } \\
\text { estropia OS }\end{array}$ \\
\hline $\mathrm{C} 2$ & M & 22 & 5 & 0.40 & Manifest nystagmus, esotropia OS \\
\hline $\mathrm{C} 3$ & M & 18 & 5 & 0.30 & $\begin{array}{l}\text { Manifest nystagmus, alternating } \\
\text { esotropia, glaucoma 0U diagnosed at } \\
\text { age } 12\end{array}$ \\
\hline C4 & $\mathrm{F}$ & 51 & 3 & 0.40 & Manifest nystagmus, squint OD \\
\hline C5 & $\mathrm{F}$ & 36 & 12 & 0.30 & $\begin{array}{l}\text { Occasional latent nystagmus, strabismus } \\
\text { surgery for esotropia, epikeratoplasty } \\
\text { at age } 1.5 \text { (no details available about } \\
\text { which eye is affected) }\end{array}$ \\
\hline $\mathrm{C} 6$ & $\mathrm{~F}$ & 34 & 17 & 0.25 & $\begin{array}{c}\text { Manifest nystagmus, esotropia } 0 \mathrm{D}, \\
\text { glaucoma } 0 \mathrm{U} \text {, astigmatism } 0 \mathrm{U}\end{array}$ \\
\hline $\mathrm{C} 7$ & $\mathrm{~F}$ & 43 & 18 & 0.17 & Manifest nystagmus \\
\hline $\mathrm{C} 8$ & $\mathrm{~F}$ & 36 & 24 & 0.10 & Manifest nystagmus, esotropia OD \\
\hline C9 & $\mathrm{F}$ & 45 & 24 & 0.40 & $\begin{array}{l}\text { Manifest nystagmus, retinal detachment } \\
\text { OU, myopia }\end{array}$ \\
\hline S1 & $\mathrm{F}$ & 26 & & & \\
\hline S2 & M & 23 & & & \\
\hline S3 & $\mathrm{F}$ & 19 & & & \\
\hline S4 & $\mathrm{F}$ & 56 & & & \\
\hline S5 & $\mathrm{F}$ & 37 & & & \\
\hline S6 & $\mathrm{F}$ & 33 & & & \\
\hline S7 & $\mathrm{F}$ & 47 & & & \\
\hline S8 & $M$ & 35 & & & \\
\hline S9 & $F$ & 45 & & & \\
\hline
\end{tabular}

$\mathrm{F}$, Female; $\mathrm{M}$, male; $0 \mathrm{~S}$, left eye; $0 \mathrm{D}$, right eye; $0 \mathrm{U}$, both eyes.

cataract-reversal individuals and normally sighted controls during speech processing in noise in two signal-to-noise ratio (SNR) conditions: high SNR $(+16.1)$ and low SNR $(-2)$.

\section{Stimuli}

Stimuli consisted of 144 monosyllabic German words, recorded from a male professional actor whose native language was German at a 16-bit resolution and a sampling rate of $48 \mathrm{kHz}$. The mean duration of the stimuli was $2268 \mathrm{~ms}$ (range, 1400-3080 ms). Stimuli were presented as lists of semantically unrelated words that were balanced for word frequency across conditions. The same lists were used for both groups. The SNR of the auditory stimuli was varied by adding multi-talker babble, so that the auditory stimuli were presented at a high $(+16.1)$ or low $(-2)$ SNR. These SNR levels were chosen on the basis of a pilot study, in which normally sighted individuals were able to correctly identify and repeat the word on average in $20 \%$ (i.e., low SNR) and 95\% (i.e., high SNR) of the trials. The maximum volume level of the auditory stimuli was adjusted so that their intensity was the same across conditions. In addition, the intensity of the auditory stimuli was individually adjusted inside the scanner and before testing to equalize the perceived loudness across participants (Collignon et al., 2011, 2013; De Martino et al., 2013, 2015; Moerel et al., 2012, 2013). This was done by increasing or decreasing the intensity of the auditory stimuli until a hearing level was reached that was both audible and comfortable for each participant.

During the presentation of the auditory stimuli, a stationary video frame showing the speaker's lips was displayed (i.e., auditory stimulation but no lip movements). In addition, a baseline condition was included consisting only of the stationary video frame showing the speaker's lips (i.e., neither auditory stimulation nor lip movements). This was done to ensure that all conditions included in the original study (Putzar et al., 2007, 2010; Guerreiro et al., 2015; comprising visual, auditory, and audiovisual stimulation conditions) were as comparable as possible, differing only with regard to the effect of interest: visual and audiovisual conditions contained lip movements, whereas a stationary video frame showing the speaker's lips (but no lip movements) was presented during the auditory (and baseline) conditions. Visual stimuli were presented on 
a screen positioned behind the head of the participant and viewed using a mirror attached to the head-coil. Auditory stimuli were presented via MRI-compatible headphones. Stimulus presentation was controlled by the software package Presentation (Neurobehavioral Systems).

\section{Task}

The task consisted of a one-back task, in which participants were required to indicate via a button press whether the word presented in the current trial was the same or not as the word presented in the previous trial. A block design was used, in which each block consisted of 12 words of the same condition, presented consecutively for $\sim 36$ s (i.e., the trial duration varied depending on the audio track length). Each block was presented six times throughout the experiment and was followed by a $15.75 \mathrm{~s}$ baseline presentation (i.e., stationary frame of the speaker's lips). The order of the conditions was randomized for each participant.

To ensure that participants kept attending to the stimuli throughout each block, the number of repetitions within each block was unpredictable (i.e., two to four repetitions). Participants responded to a stimulus repetition with their right index finger and to a nonrepetition with the right middle finger. Participants were asked to respond as accurately as possible, as well as before the end of the respective audio track.

\section{fMRI data acquisition}

Images were acquired on a 3 Tesla Siemens Magnetom TRIO scanner equipped with a standard head coil at the University Medical Center Hamburg-Eppendorf. Anatomical T1-weighted volumes covering the whole brain were obtained using a $3 \mathrm{D}$ fast, low-angle shot sequence [repetition time (TR), $15 \mathrm{~ms}$; echo time (TE), $4.92 \mathrm{~ms}$; flip angle (FA), $25^{\circ}$; 192 sagittal slices; slice thickness, $1 \mathrm{~mm}$; slice gap, $0 \mathrm{~mm}$; voxel dimensions, $1 \times 1 \times 1 \mathrm{~mm}$; matrix size, $256 \times 256]$. Functional T2*weighted images were acquired with a spin echo/echo planar imaging sequence (TR, $2250 \mathrm{~ms}$; TE, $25 \mathrm{~ms}$; FA, $80^{\circ}$; 37 axial slices; slice thickness, $2 \mathrm{~mm}$; slice gap, $1 \mathrm{~mm}$; in-plane resolution, $3 \times 3 \mathrm{~mm}$; field of view, $192 \times 192 \mathrm{~mm}$; matrix size, $64 \times 64)$. Foam cushions and padding were used to stabilize head position and minimize head movements.

\section{fMRI data analysis}

Functional and anatomical data were analyzed using BrainVoyager QX 2.8 (Brain Innovation). The first two images of each functional run were discarded to account for the initial magnetization in equilibrium. Functional preprocessing consisted of slice scan-time correction, headmotion correction, and temporal high-pass filtering (removing linear trends and frequencies $<3$ cycles/run). If head movements in a run exceeded $3 \mathrm{~mm} /{ }^{\circ}$ in any direction, the respective run was excluded from additional analysis. Four high SNR blocks and two low SNR blocks in the group of normally sighted controls, as well as five high SNR blocks and four low SNR blocks in the group of cataract-reversal individuals, were excluded because of head movements exceeding this criterion or because of technical problems. To improve the alignment of data across participants for whole-brain group analyses, the data were spatially smoothed with a Gaussian kernel of $4 \mathrm{~mm}$ full-width half-maximum.

Individual anatomical images were transformed into Talairach space and segmented at the gray/white matter boundary, allowing for cortical surface reconstruction of each individual participant's brain hemispheres. Cortex-based alignment was then performed to further improve the spatial correspondence mapping between participants' brains beyond Talairach space matching. The reconstructed cortical surfaces were transformed into a spherical representation that was subjected to nonrigid alignment to a selected target brain sphere (i.e., the atlas brain provided by BrainVoyager QX) based on the gyral/sulcal folding pattern.
The aligned cortical surfaces were used to create an average cortex representation of all participants. Functional images were coregistered to the anatomical images and transformed into surface space.

Region of interest analysis. Because we were interested in comparing the response profile of specific sensory cortical regions between cataractreversal individuals and normally sighted controls, and to increase statistical power, we used a region of interest (ROI) approach (Poldrack, 2007). The following ROIs were included: posterior superior temporal gyrus (Brodmann areas 41 and 42), calcarine sulcus, and fusiform gyrus (Fig. 1). These regions were selected on the basis of their involvement in speech processing in noise in the normally sighted population (i.e., posterior superior temporal gyrus; Wong et al., 2008), as well as on the basis of their involvement in speech processing in blind individuals (i.e., calcarine sulcus and fusiform gyrus; Hertrich et al., 2009; Dietrich et al., 2013). To avoid circularity (Kriegeskorte et al., 2009), we used an anatomical criterion for ROI definition. Specifically, ROIs were automatically labeled for each participant after aligning their cortical surface reconstructions to the atlas brain provided by BrainVoyager QX. This procedure enables an accurate remapping of predefined ROIs from the atlas brain provided by BrainVoyager QX to each individual participant's cortex by making use of the cortical curvature information of the individual and the atlas brain.

The data were analyzed with a single-subject general linear model comprising two predictors of interest: high SNR and low SNR. Movement parameters, as well as task instruction and a short rest interval at the beginning and at the end of each run (lasting 4.5 and $9 \mathrm{~s}$, respectively), were treated as confound predictors. The stationary frame of the speaker's lips was implicitly modeled as baseline in the design.

The average $\beta$ values of each experimental condition were calculated within each ROI and submitted to a repeated-measures ANOVA with group (two levels: cataract-reversal individuals and normally sighted controls) as a between-group factor and hemisphere (two levels: left and right) and SNR (two levels: high and low) as within-group factors. $\beta$ values quantify the contribution of each predictor to the observed data within an ROI, providing an indication of the level of activation for each condition within the ROI (Huettel et al., 2009). Specifically, large positive (or negative) $\beta$ values typically indicate that the ROI exhibits strong activation (or deactivation) during the modeled experimental condition relative to baseline, whereas small $\beta$ values indicate that the ROI exhibits little or no difference in activation during the modeled experimental condition compared with baseline. 

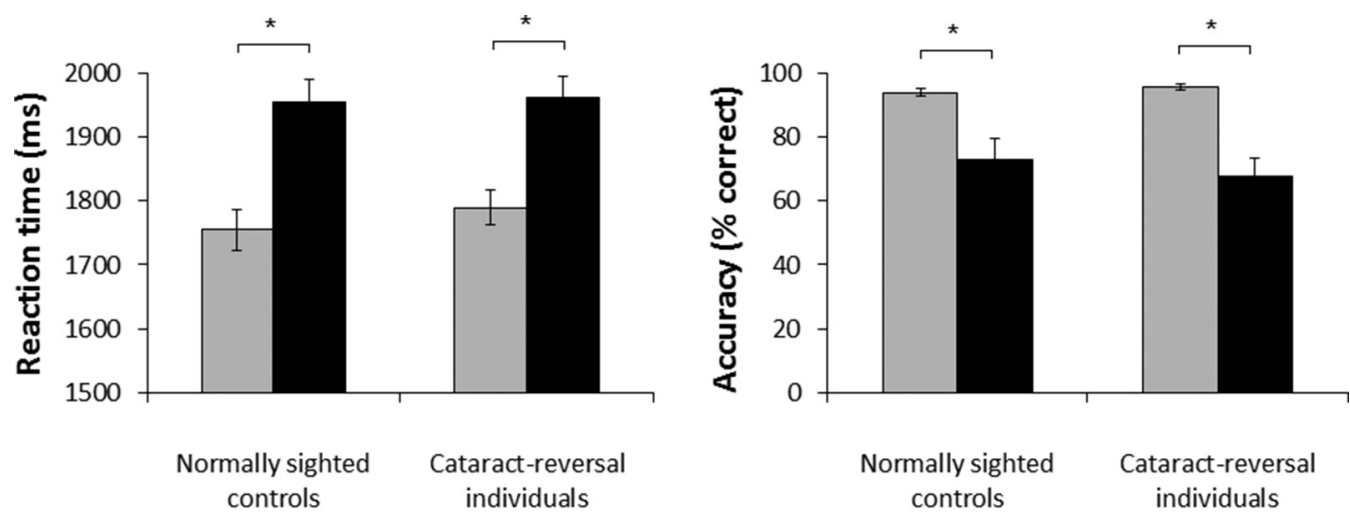

口High SNR

- Low SNR

Figure 2. Behavioral performance. Left, Mean reaction time and SEs for normally sighted controls and cataract-reversal individuals. Right, Mean accuracy and SEs of the mean for normally sighted controls and cataract-reversal individuals. ${ }^{*} p<0.050$.

Single-subject analyses were subsequently performed to assess the consistency of effects across individuals. Single-subject analyses additionally allow us to exclude the role of any potential differences in hearing thresholds across individuals (although unlikely; Starlinger and Niemeyer, 1981) because hearing thresholds are the same within a participant. In these individual analyses, we compared activation in each ROI in response to low and high SNR conditions (low SNR > high SNR) in each participant, separately by hemisphere.

Whole-brain analysis. To assess whether eventual group differences in the selected ROIs could be compensated by (or generalized to) other brain regions not included in the ROI analysis and to demonstrate that the ROI results are independent of the criterion used for ROI selection (Kriegeskorte et al., 2009), we additionally performed a whole-brain analysis.

The data were analyzed with a multi-subject general linear model comprising two predictors of interest separated for each subject: low SNR and high SNR. Movement parameters, as well as task instruction and a short rest interval at the beginning and end of each run (lasting 4.5 and $9 \mathrm{~s}$, respectively), were treated as confound predictors. The still frame of the speaker's lips was implicitly modeled as baseline in the design. To optimize the preservation of differences between individual effect sizes, each run was normalized in terms of percentage signal change (Stilla et al., 2008).

We performed whole-brain analyses within and between groups using a random-effects ANOVA, with group (two levels: cataract-reversal individuals and normally sighted controls) as a between-group factor and SNR (two levels: high and low) as a within-group factor. In a first step, we compared brain responses to low SNR with brain responses to high SNR (low SNR $>$ high SNR) separately by group to assess the cortical mechanisms of speech perception in noise within each group. In a second step, we tested for group differences in this effect with a group (sighted controls $>$ cataract individuals) $\times$ SNR (low SNR $>$ high SNR) interaction analysis. In a final step, we compared brain responses between groups (sighted controls $>$ cataract individuals) separately by SNR condition to assess group differences in the cortical mechanisms of speech perception in noise at each SNR level. The voxel threshold for statistical significance was set to $p<0.01$ uncorrected, and the statistical contrast maps were corrected for multiple comparisons using cluster-size thresholding (Forman et al., 1995; Goebel et al., 2006), with a cluster-level false-positive rate of $\alpha=0.05$.

\section{Behavioral analysis}

Reaction times pertaining to correct responses and percentage of correct responses were submitted to a repeated-measures ANOVA with group (two levels: cataract-reversal individuals and normally sighted controls) as a between-group factor and SNR (two levels: high and low) as a within- group factor. Because of technical problems with the response device, behavioral data were not available for two normally sighted participants.

\section{Results}

\section{Behavioral results}

\section{Reaction time}

There was no effect of group $\left(F_{(1,14)}=0.29, p=0.596, \eta^{2}=0.02\right)$ such that overall reaction time did not differ between cataractreversal individuals and normally sighted controls. The effect of SNR was significant $\left(F_{(1,14)}=84.19, p<0.001, \eta^{2}=0.85\right)$, indicating that responses were faster in the high SNR condition than in the low SNR condition. Group did not interact with this effect $\left(F_{(1,14)}=0.42, p=0.526, \eta^{2}=0.004\right)$, revealing that it did not differ between cataract-reversal individuals and normally sighted controls (Fig. 2, left).

\section{Accuracy}

There was no effect of group $\left(F_{(1,14)}=0.13, p=0.720, \eta^{2}=\right.$ $0.01)$, indicating that overall accuracy did not differ between cataract-reversal individuals and normally sighted controls. The effect of SNR was significant $\left(F_{(1,14)}=37.80, p<0.001, \eta^{2}=\right.$ 0.72 ), indicating that responses were more accurate in the high SNR condition than in the low SNR condition. Group did not interact with this effect $\left(F_{(1,14)}=0.72, p=0.412, \eta^{2}=0.01\right)$, revealing that it did not differ between cataract-reversal individuals and normally sighted controls (Fig. 2, right).

\section{Imaging results}

ROI results

Posterior superior temporal gyrus. There was no effect of group $\left(F_{(1,16)}=0.58, p=0.456, \eta^{2}=0.04\right)$ such that overall activation in the posterior superior temporal gyrus did not differ between cataract-reversal individuals and normally sighted controls. The effect of SNR $\left(F_{(1,16)}=7.09, p=0.017, \eta^{2}=0.11\right)$ and the interaction between SNR and group $\left(F_{(1,16)}=5.26, p=0.036, \eta^{2}\right.$ $=0.08$ ) were both significant, revealing that the effect of SNR differed across groups (Fig. 3, left). In the group of normally sighted controls, activation in the posterior superior temporal gyrus was higher in the low SNR condition than in the high SNR condition $\left(F_{(1,8)}=9.21, p=0.016, \eta^{2}=0.30\right)$; in contrast, in the group of cataract-reversal individuals, activation in the posterior superior temporal gyrus did not differ across SNR conditions 
Posterior superior temporal gyrus

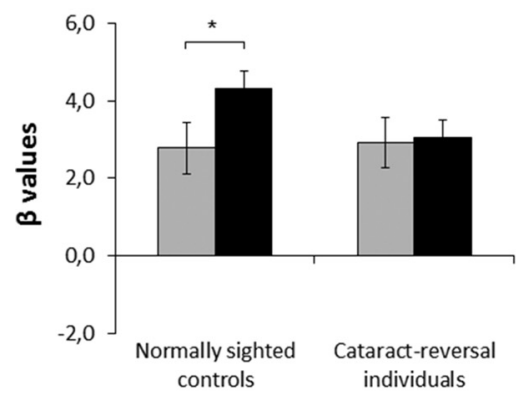

Calcarine sulcus

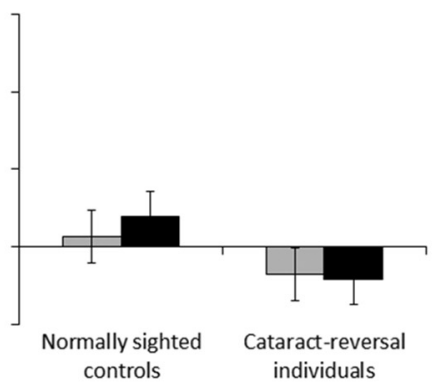

$\square$ High SNR
Fusiform gyrus

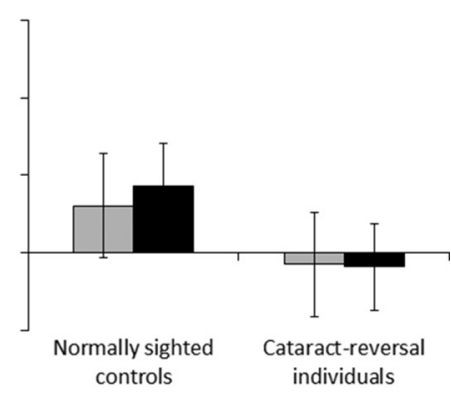

- Low SNR

Figure 3. Mean $\beta$ values and SEs in the ROls for normally sighted controls and cataract-reversal individuals. Left, Posterior superior temporal gyrus; middle, calcarine sulcus; right, fusiform gyrus. ${ }^{*} p<0.050$.

Table 2. Differences in cortical responses between low and high SNR in each ROI in the single-subject analyses

\begin{tabular}{|c|c|c|c|c|c|c|}
\hline & \multicolumn{2}{|c|}{ Posterior superior temporal gyrus } & \multicolumn{2}{|c|}{ Calcarine sulcus } & \multicolumn{2}{|c|}{ Fusiform gyrus } \\
\hline & $\mathrm{LH}$ & $\mathrm{RH}$ & LH & $\mathrm{RH}$ & $\mathrm{LH}$ & $\mathrm{RH}$ \\
\hline S1 & 0.19 & 0.23 & -0.74 & -0.48 & -0.43 & 0.20 \\
\hline $\mathrm{S} 2$ & -0.85 & $2.25^{*}$ & 1.17 & 1.59 & 0.84 & 1.55 \\
\hline S3 & 1.04 & -0.09 & -1.56 & -0.15 & -0.39 & -0.85 \\
\hline S4 & 1.10 & $3.12^{* *}$ & 1.07 & 0.46 & 0.07 & 0.19 \\
\hline S5 & $5.31^{* * *}$ & $5.83^{* * *}$ & 1.14 & 1.01 & 1.06 & 0.72 \\
\hline S6 & 0.01 & -0.98 & 0.02 & 0.48 & -0.18 & $2.24^{*}$ \\
\hline S7 & $1.97^{*}$ & 1.79 & -1.58 & -1.14 & -1.21 & -1.46 \\
\hline S8 & $2.70^{* *}$ & $4.81^{* * *}$ & -0.45 & 0.16 & -0.96 & 0.18 \\
\hline S9 & $5.44^{* * *}$ & $6.28 * * *$ & $2.85^{* *}$ & $3.91^{* * *}$ & 1.21 & $2.96^{* *}$ \\
\hline C1 & 1.86 & 1.02 & 0.60 & 0.57 & 0.48 & -0.35 \\
\hline $\mathrm{C} 2$ & 1.76 & $2.58^{*}$ & 1.00 & 0.83 & 0.43 & 1.13 \\
\hline C 3 & -1.63 & -0.48 & 0.46 & 1.94 & 1.18 & $2.32^{*}$ \\
\hline C4 & -0.16 & 0.52 & -0.88 & -1.79 & -1.00 & -1.48 \\
\hline$C 5$ & -0.77 & 0.48 & $-3.47^{* * *}$ & $-2.37^{*}$ & 0.31 & 0.21 \\
\hline C6 & -0.05 & 0.55 & 1.84 & 0.54 & -1.58 & -0.89 \\
\hline C7 & $-2.90^{* *}$ & $-2.75^{* *}$ & 0.810 & 0.86 & 1.36 & 0.85 \\
\hline C8 & $-2.10^{*}$ & -0.06 & 0.44 & -0.96 & -1.01 & -0.84 \\
\hline C9 & 1.53 & 0.15 & -1.33 & $-1.97^{*}$ & -0.71 & 0.36 \\
\hline
\end{tabular}

Positive $t$ values indicate higher activation during low SNR; negative $t$ values indicate lower activation during low SNR. LH, Left hemisphere; RH, right hemisphere.

${ }^{*} p<0.050$.

${ }^{* *} p<0.010$.

${ }^{* * *} p<0.001$.

$\left(F_{(1,8)}=0.10, p=0.757, \eta^{2}=0.003\right)$. No other effects reached significance ( $p$ values $>0.050$ ).

Single-subject analysis confirmed the pattern of results obtained with the group analysis (Table 2, left two columns). In the normally sighted control group, activation in the posterior superior temporal gyrus was significantly higher in the low SNR condition than in the high SNR condition in both hemispheres of three participants (S5, S8, and S9), in the right hemisphere of two participants (S2 and S4), and in the left hemisphere of one participant (S7). In contrast, in the cataract-reversal group, activation in the posterior superior temporal gyrus was significantly higher in the low SNR condition than in the high SNR condition in the right hemisphere of only one participant (C2). Thus, whereas the majority of normally sighted individuals $(n=6)$ exhibited significantly higher activation in the posterior superior temporal gyrus in the low SNR condition than in the high SNR condition in at least one of the hemispheres, only one cataractreversal individual showed this pattern. Because auditory stimuli were presented at the same intensity within each participant and hearing acuity is constant within each participant, differences between auditory conditions (or lack thereof) in a given participant cannot be accounted for by differences in hearing acuity.

Calcarine sulcus. There was no effect of group $\left(F_{(1,16)}=2.20\right.$, $p=0.157, \eta^{2}=0.12$ ), indicating that overall activation in the calcarine sulcus did not differ between cataract-reversal individuals and normally sighted controls. The effect of SNR $\left(F_{(1,16)}=\right.$ $\left.0.38, p=0.544, \eta^{2}=0.02\right)$ and the interaction between SNR and group $\left(F_{(1,16)}=1.11, p=0.308, \eta^{2}=0.05\right)$ were both nonsignificant, revealing that activation in the calcarine sulcus did not differ across SNR conditions in either group (Fig. 3, middle). No other effects reached significance ( $p$ values $>0.050$ ).

Single-subject analysis confirmed the pattern of results obtained with the group analysis (Table 2, middle two columns). In the normally sighted control group, activation in the calcarine sulcus was significantly higher in the low SNR condition than in the high SNR condition in both hemispheres of one participant (S9), whereas in the remaining cases, it did not significantly differ across SNR conditions. In the cataract-reversal group, if anything, activation in the calcarine sulcus was significantly lower in the low SNR condition than in the high SNR condition in both hemispheres of one participant (C5) and in the right hemisphere of one other participant (C9), whereas in the remaining cases, it did not differ significantly across SNR conditions. Thus, the majority of normally sighted individuals $(n=8)$ and cataractreversal individuals $(n=7)$ showed no significant difference in calcarine sulcus activation across SNR conditions.

Fusiform gyrus. There was no effect of group $\left(F_{(1,16)}=1.16\right.$, $p=0.297, \eta^{2}=0.07$ ) such that overall activation in the fusiform gyrus did not differ between cataract-reversal individuals and normally sighted controls. The effect of $\operatorname{SNR}\left(F_{(1,16)}=0.17, p=\right.$ $\left.0.688, \eta^{2}=0.003\right)$ and the interaction between SNR and group $\left(F_{(1,16)}=0.25, p=0.621, \eta^{2}=0.005\right)$ were both nonsignificant, indicating that activation in the fusiform gyrus did not differ across SNR conditions in either group (Fig. 3, right). No other effects reached significance ( $p$ values $>0.050$ ).

Single-subject analysis confirmed the pattern of results obtained with the group analysis (Table 2, right two columns). Activation in the fusiform gyrus was significantly higher in the low SNR condition than in the high SNR condition in the right hemisphere of two normally sighted participants (S6 and S9) and in the right hemisphere of one cataract-reversal participant (C3), whereas in the remaining cases, it did not significantly differ 


\section{A}

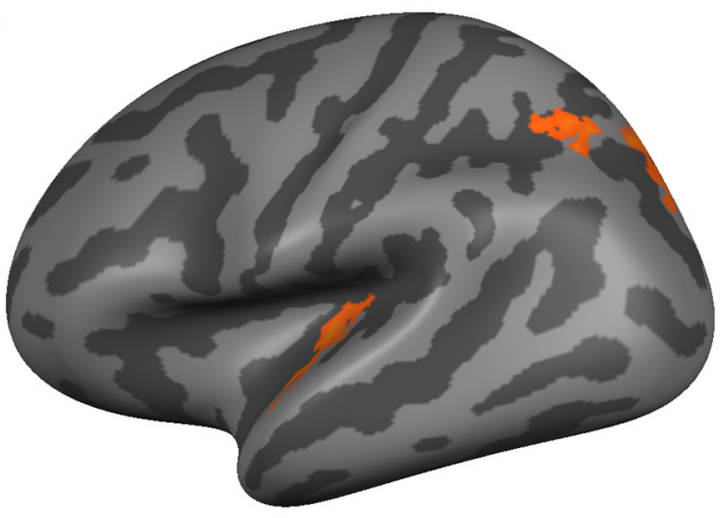

B

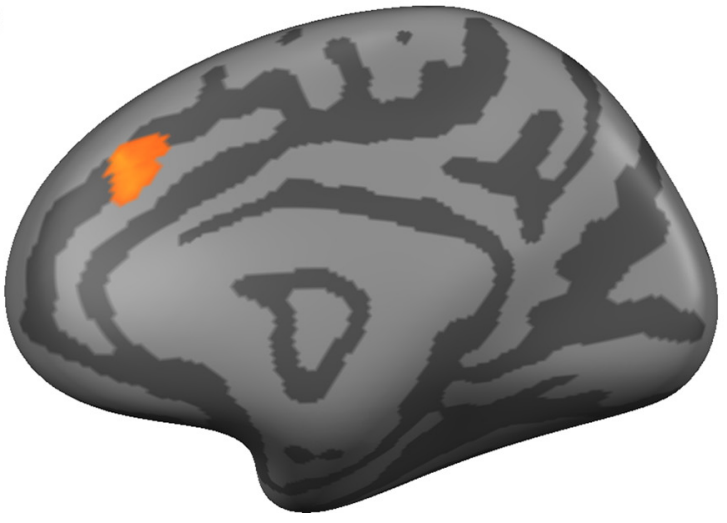

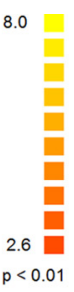

8.0 $p<0.01$

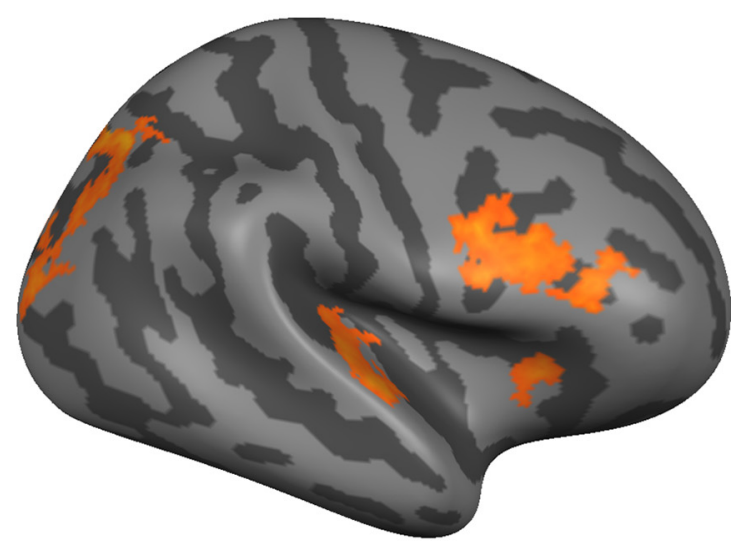

8.0

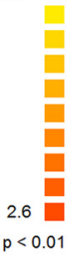

$<0.01$

Figure 4. Activations obtained when comparing brain responses to low SNR and brain responses to high SNR (low SNR $>$ high SNR) in the group of normally sighted controls $(\boldsymbol{A})$ and the group of cataract-reversal individuals $(\boldsymbol{B})$, overlaid on the average cortex reconstruction of all participants.

across SNR conditions. Thus, the majority of normally sighted individuals $(n=7)$ and cataract-reversal individuals $(n=8)$ showed no significant difference in fusiform gyrus activation across SNR conditions.

\section{Whole-brain results}

The results of the ROI analysis suggest that cataract-reversal individuals, unlike normally sighted controls, do not show auditory cortex modulation in response to low SNR auditory stimuli. To assess whether this lack of auditory cortex modulation may be compensated by increased activation in other brain regions not included in the ROI analysis and to demonstrate that the ROI results are independent of the criterion used for ROI selection (Kriegeskorte et al., 2009), we additionally performed a wholebrain analysis.

In the group of normally sighted controls, the contrast between brain responses to low SNR and brain responses to high SNR (low SNR > high SNR) revealed a greater recruitment of a number of areas in response to low SNR stimulation, including the posterior superior temporal gyrus bilaterally, the middle frontal gyrus bilaterally, the superior parietal lobule bilaterally, the right inferior frontal gyrus, the right insula, the right cuneus, and the left precuneus (Fig. 4, top; Table 3, Normally sighted controls). There were no areas of increased activation in the opposite contrast (high SNR $>$ low SNR). In the group of cataractreversal individuals, the contrast between brain responses to low SNR and brain responses to high SNR (low SNR > high SNR) revealed a single focal area of increased activation in response to low SNR auditory stimulation, which was located within the right
Table 3. Differences in brain responses between low and high SNR conditions in normally sighted controls and cataract-reversal individuals

\begin{tabular}{lcrrrr}
\hline Region & $\begin{array}{l}\text { Cluster } \\
\text { size }\end{array}$ & $x$ & $y$ & $z$ & $t$ \\
\hline Normally sighted controls & & & & & \\
$\quad$ Right superior parietal lobule & 496 & 28 & -60 & 44 & 5.45 \\
$\quad$ Right inferior frontal gyrus & 414 & 43 & 3 & 31 & 4.73 \\
$\quad$ Right superior temporal gyrus & 274 & 54 & -11 & 7 & 4.66 \\
$\quad$ Right cuneus & 49 & 15 & -86 & 21 & 4.34 \\
$\quad$ Left precuneus & 164 & -14 & -71 & 44 & 4.24 \\
$\quad$ Right insula & 89 & 31 & 15 & 8 & 4.17 \\
$\quad$ Left superior temporal gyrus & 167 & -36 & -21 & 6 & 4.05 \\
$\quad$ Right middle frontal gyrus & 100 & 47 & 26 & 20 & 3.96 \\
$\quad$ Left superior parietal lobule & 109 & -24 & -58 & 45 & 3.86 \\
Cataract-reversal individuals & & & & & \\
$\quad$ Right anterior cingulate gyrus & 149 & 8 & 25 & 38 & 4.14 \\
\hline$x, y, z$ are Talairach coordinates of peak voxel; $t=$ peak $t$ value; $p<0.050$ corrected at the cluster level.
\end{tabular}

anterior cingulate gyrus (Fig. 4, bottom; Table 3, Cataractreversal individuals). There were no areas of increased activation in the opposite contrast (high SNR $>$ low SNR).

Although the group (sighted controls $>$ cataract patients) $\times$ SNR (low SNR > high SNR) interaction analysis did not reveal significant differences between groups-likely because of the relatively low number of participants ( $n=9$ per group) for such an analysis, which is known to inflate type II error in group comparisons (Huettel et al., 2009) — planned comparisons revealed clear group differences that differed across SNR levels. Specifically, during high SNR auditory stimulation, there were no differences 

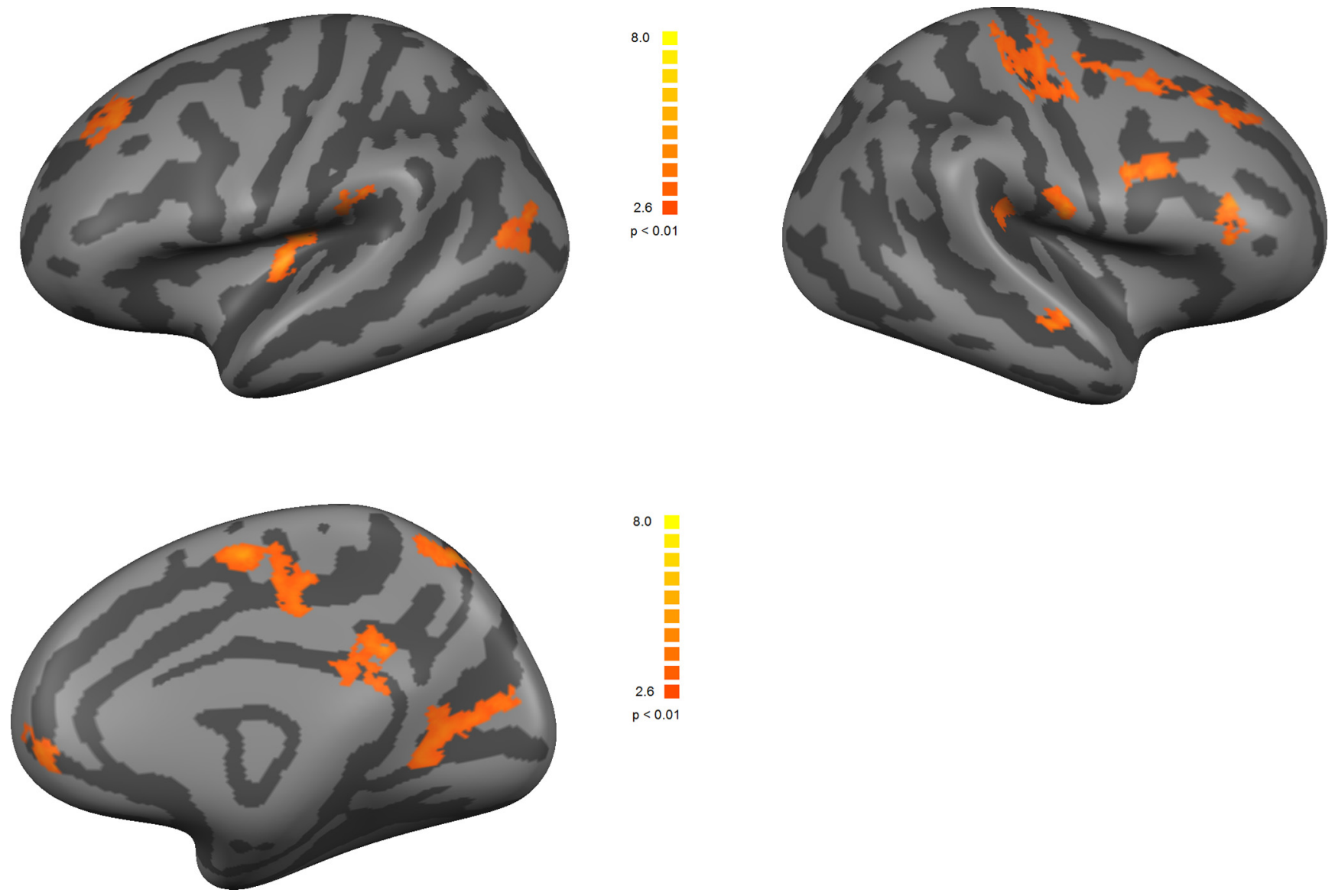

Figure 5. Activations obtained when comparing brain responses of normally sighted controls with brain responses of cataract-reversal individuals (sighted controls $>$ cataract individuals) during low SNR auditory stimulation, overlaid on the average cortex reconstruction of all participants.

in brain activation between groups (sighted controls $>$ cataract individuals; cataract individuals $>$ sighted controls). However, during low SNR auditory stimulation, the contrast between brain responses of normally sighted controls and brain responses of cataract-reversal individuals (sighted controls $>$ cataract individuals) revealed a greater recruitment of posterior superior temporal areas bilaterally, as well as of areas partially associated with the frontoparietal attentional network (e.g., middle frontal gyrus bilaterally, right medial frontal gyrus, right precuneus, left inferior parietal lobule, and right insula) in normally sighted controls (Fig. 5; Table 4). The opposite contrast (cataract individuals $>$ sighted controls) revealed no areas of increased activation in cataract-reversal individuals during low SNR stimulation.

\section{Correlations}

Brain activation and visual deprivation

To explore the relationship between brain activation and duration of visual deprivation (i.e., time from birth until cataract surgery), we performed two-tailed Kendall's rank correlations between $\beta$ values from each ROI and duration of visual deprivation in the group of cataract-reversal individuals. Because there were no significant effects involving the factors hemisphere or SNR in any of the ROIs considered in the group of cataractreversal individuals, $\beta$ values were collapsed across hemispheres and SNR levels. This analysis yielded no significant correlation between duration of visual deprivation and activation in the calcarine sulcus $(\tau=0.06, p=0.833)$, fusiform
Table 4. Differences in brain responses between normally sighted controls and cataract-reversal individuals during low SNR auditory stimulation

\begin{tabular}{lrrrrr}
\hline Region & $\begin{array}{l}\text { Cluster } \\
\text { size }\end{array}$ & \multicolumn{1}{c}{ x } & & $z$ & $t$ \\
\hline Normally sighted controls & & & & & \\
$\quad$ Left superior temporal gyrus & 177 & -33 & -18 & 7 & 4.45 \\
Left middle occipital gyrus & 123 & -37 & -71 & 20 & 3.64 \\
Left middle frontal gyrus & 153 & -23 & 26 & 39 & 3.96 \\
Left inferior parietal lobule & 50 & -56 & -30 & 28 & 3.95 \\
Right insula & 54 & 42 & -28 & 25 & 3.49 \\
Right postcentral gyrus & 60 & 59 & -13 & 21 & 4.38 \\
Right posterior cingulate gyrus & 268 & 22 & -52 & 10 & 3.67 \\
Right middle frontal gyrus & 111 & 28 & 2 & 50 & 3.82 \\
Right medial frontal gyrus & 248 & 6 & -7 & 52 & 4.51 \\
Right anterior cingulate gyrus & 162 & 7 & -38 & 31 & 4.24 \\
Right precuneus & 94 & 10 & -56 & 51 & 4.48 \\
Right middle frontal gyrus & 52 & 42 & 27 & 24 & 3.99 \\
Right inferior frontal gyrus & 103 & 39 & 4 & 29 & 4.04 \\
Right superior temporal gyrus & 36 & 47 & -13 & -4 & 4.10 \\
Right precentral gyrus & 314 & 33 & -22 & 49 & 3.67 \\
Right middle frontal gyrus & 106 & 26 & 15 & 47 & 4.09 \\
Right anterior cingulate gyrus & 52 & 12 & 44 & 3 & 3.95 \\
\hline
\end{tabular}

$x, y, z$ are Talairach coordinates of peak voxel; $t=$ peak $t$ value; $p<0.050$ corrected at the cluster level.

gyrus $(\tau=0.06, p=0.833)$, or posterior superior temporal gyrus $(\tau=0.17, p=0.527)$

Brain activation and sight recovery

The study by Dormal et al. (2015) suggested that crossmodal plasticity in sight-recovery individuals decays as time from sur- 

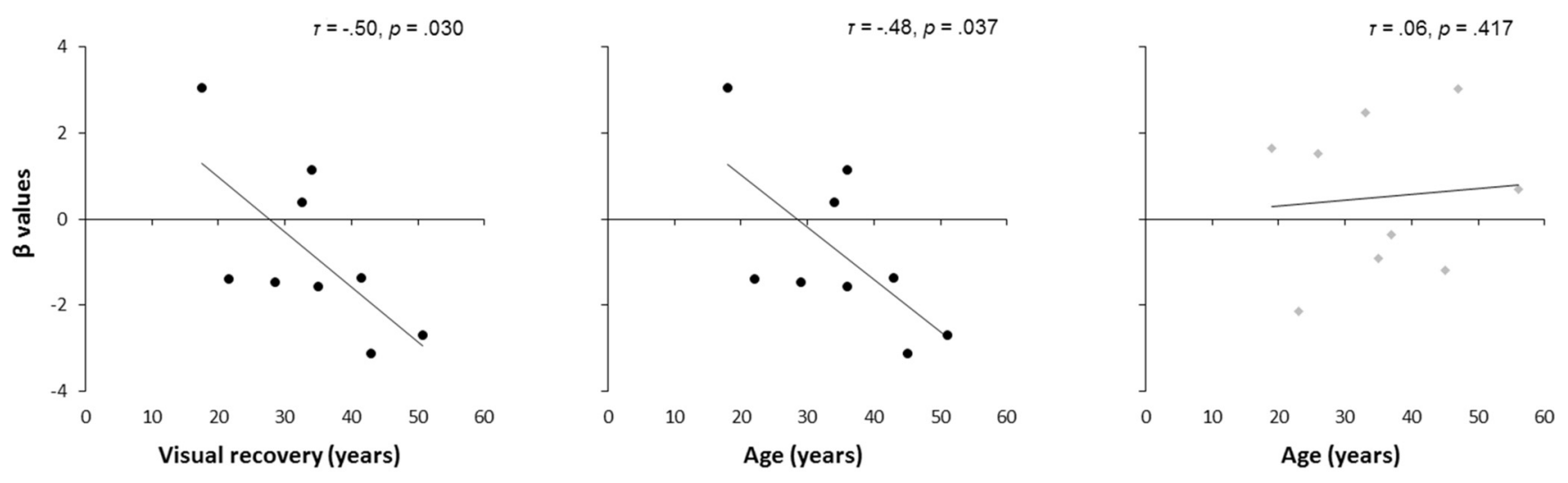

- Cataract-reversal individuals
- Normally sighted controls

Figure 6. Left, Correlation between $\beta$ values in the calcarine sulcus (collapsed across hemisphere and SNR) and duration of visual recovery in cataract-reversal individuals. Middle, Correlation between $\beta$ values in the calcarine sulcus (collapsed across hemisphere and SNR) and age in cataract-reversal individuals. Right, Correlation between $\beta$ values in the calcarine sulcus (collapsed across hemisphere and SNR) and age in normally sighted individuals.

gery elapses. To further probe the possibility that crossmodal plasticity reverts over time, we performed one-tailed Kendall's rank correlations between $\beta$ values from visual ROIs (collapsed across hemispheres and SNR) and the duration of sight recovery (i.e., time since cataract surgery) in the group of cataract-reversal individuals. This analysis revealed a significant negative correlation between duration of sight recovery and activation in the calcarine sulcus ( $\tau=-0.50, p=0.030)$, suggesting that the longer the period of sight recovery, the lower the primary visual cortex activation during auditory processing (Fig. 6, left). The correlation between duration of sight recovery and activation in the fusiform gyrus did not reach significance $(\tau=0.17, p=$ 0.527).

Because those individuals with longer periods of visual recovery are also those with older ages, we deemed it necessary to exclude the possibility that the correlation above could reflect an aging effect. Therefore, we performed a one-tailed, partial Kendall's rank correlation between $\beta$ values from the calcarine sulcus (collapsed across hemispheres and SNR) and the duration of sight recovery (i.e., time since cataract surgery), while controlling for the effect of age. Although the correlation reported above was no longer significant when age was controlled for $\left(\tau_{p}=-0.17\right.$, $p=0.281$ ), this is unlikely to be an aging effect because no correlation emerged between age and brain activation in the calcarine sulcus in the group of normally sighted controls $(\tau=0.06$, $p=0.417)$. In fact, such a correlation was only present in the group of cataract-reversal individuals $(\tau=-0.48, p=0.037)$. If this were a true aging effect, then the same correlation should have been observed in the group of normally sighted controls (Fig. 6, middle and right).

\section{Brain activation and visual acuity}

Finally, because the cataract-reversal individuals in the present study had lower visual acuity than the normally sighted control participants, who had normal or corrected-to-normal vision, we performed two-tailed Kendall's rank correlations between $\beta$ values from each ROI (collapsed across hemispheres and SNR) and visual acuity in the group of cataract-reversal individuals. This analysis yielded no significant correlations ( $p$ values $>0.050$ ), rendering visual acuity as a possible confounding effect unlikely in the present study. To the extent that this possibility can be ruled out, the differences in auditory cortical processing between groups observed in the present study appear to be better accounted for by the lack of visual input within the first few months after birth.

\section{Discussion}

The goal of this study was to investigate the effect of a transient phase of congenital visual deprivation on the neural bases of auditory processing. To this end, we compared auditory and visual cortical responses of cataract-reversal individuals and normally sighted controls with auditory speech presented in noise at different SNR levels while participants underwent fMRI. Although there were no behavioral group differences (Putzar et al., 2010, 2012), there were differences between groups in the neural substrates of auditory processing, particularly within the auditory cortex. We discuss these group differences below.

In line with previous research in the normally sighted population (Wong et al., 2008, 2009), normally sighted individuals showed higher activation in the posterior superior temporal gyrus as SNR decreased. In contrast, cataract-reversal individuals maintained a comparable level of auditory cortex activation across SNR conditions. The results of the whole-brain analysis replicated and extended these findings. In fact, not only did the normally sighted group exhibit a greater recruitment of posterior superior temporal areas in response to low compared with high SNR but also a greater recruitment of frontal and parietal areas known to be involved in attentional control (Owen et al., 2005; Ptak, 2012), such as the middle frontal gyrus and the superior parietal lobule bilaterally, the right inferior frontal gyrus, and the left precuneus. In contrast, the cataract-reversal group revealed only a single area of increased activation in response to low compared with high SNR, located within the right anterior cingulate cortex. Furthermore, although the groups did not show any differences in brain activation during high SNR, during low SNR, normally sighted controls displayed a greater recruitment of posterior superior temporal areas and of a number of frontoparietal areas, including the middle frontal gyrus bilaterally, the right medial frontal gyrus, the right precuneus, the left inferior parietal lobule, and the right insula, relative to cataract-reversal individuals. The recruitment of frontal and parietal areas in response to low SNR in the normally sighted group strongly suggests that performance of the same one-back task with low (rather than 
high) SNR auditory stimuli posed increased attentional demands in this group but not in the group of cataract-reversal individuals. Importantly, despite the lack of auditory cortex modulation in the cataract-reversal group and despite the lack of increased recruitment of frontoparietal areas involved in attentional control, the behavioral performance of cataract-reversal individuals did not differ from that of normally sighted controls. These observations suggest that, although there are no overt group differences during speech processing in noise (Putzar et al., 2007), cataractreversal individuals exhibit more efficient cortical processing of speech in particularly noisy conditions (i.e., low SNR).

These findings are reminiscent of studies performed in darkreared or lid-sutured animals, in which visually deprived animals show comparable or improved abilities in the remaining senses relative to normally sighted animals (Crémieux et al., 1986; Rauschecker and Kniepert, 1994; Toldi et al., 1994; King and Parsons, 1999), along with an expansion of the cortical areas representing the intact modalities (Bronchti et al., 1992; Rauschecker et al., 1992; Rauschecker and Korte, 1993; Toldi et al., 1994) and a sharper spatial tuning in auditory neurons (Korte and Rauschecker, 1993). Correspondingly, studies in permanently blind humans have provided evidence for intramodal plasticity in the auditory system (Kujala et al., 1992; Röder et al., 1996). In particular, an increasing number of fMRI studies have started to reveal lower auditory cortex activation during auditory processing in blind individuals relative to sighted controls (Gougoux et al., 2009; Stevens and Weaver, 2009; Watkins et al., 2013; Hölig et al., 2014a), suggesting more efficient cortical processing of auditory stimuli within the early stages of the auditory hierarchy (Röder et al., 1996; Stevens and Weaver, 2009). The present study extends these findings by demonstrating that such intramodal changes (i.e., lower auditory cortex activation during auditory processing) are observed in individuals who experienced visual depri-

vation within the first few months after birth. Importantly, although cataract-reversal individuals had lower visual acuity than normally sighted controls, there was no correlation between auditory cortical activity and visual acuity in the cataract-reversal group, arguing against the possibility that intramodal plasticity could be explained on the basis of their reduced visual acuity. Likewise, although we did not formally test for differences in hearing thresholds between groups, the present results cannot be accounted for by potential differences in hearing acuity across individuals because the SNR manipulation was orthogonal to the intensity of the stimuli. Moreover, the group-level results were replicated in single-subject analyses. Individual analyses allow us to rule out potential differences in hearing thresholds across individuals because auditory stimuli were presented at the same intensity within each participant and hearing acuity was the same within each participant. Thus, it seems justified to conclude that a transient period of congenital visual deprivation might be sufficient to induce intramodal plastic changes within the auditory cortex of cataract-reversal individuals.

Another goal of the present study was to evaluate the presence of residual crossmodal plasticity during auditory processing in cataract-reversal individuals. Previous studies have shown that crossmodal auditory responses coexist with regained visual responses within the visual cortex of sight-recovery individuals (Saenz et al., 2008; Dormal et al., 2015) but decrease over time after sight restoration (Dormal et al., 2015). However, individuals in these studies were blind for an extended period of time (i.e., 35-43 years), which has not always started at birth. Here we attempted to further isolate the role of early visual experience (or lack thereof) on crossmodal responses after sight recovery. To this end, we compared visual cortical responses of cataractreversal individuals and normally sighted controls to the presentation of auditory speech in two visual areas shown previously to be involved in speech processing in blind individuals, namely calcarine sulcus and fusiform gyrus (Röder et al., 2002; Hertrich et al., 2009; Bedny et al., 2011, 2012; Dietrich et al., 2013). We found no evidence for crossmodal plasticity during auditory speech processing at the group level in the present study. In fact, there were no differences in visual cortical responses between cataract-reversal individuals and normally sighted controls in either SNR condition.

The lack of crossmodal responses in the present study suggests that crossmodal plasticity takes longer to anchor in the brain than the duration of visual deprivation in the investigated sample. This would explain why crossmodal plasticity was observed in patients whose period of visual deprivation lasted 35-43 years (Saenz et al., 2008; Dormal et al., 2015) but not in the present sample whose period of visual deprivation from birth lasted only up to 2 years. Alternatively, crossmodal plasticity may have regressed (Dormal et al., 2015) such that it dissipated by the time of testing. This would account for the crossmodal plasticity observed in patients whose period of visual recovery lasted 7 months to 10 years after surgery (Saenz et al., 2008; Dormal et al., 2015) but not in the present sample, whose period of visual recovery lasted 18-51 years. The results of the correlational analyses support the latter hypothesis. Indeed, although no correlation emerged between duration of visual deprivation and visual cortex activation, there was a significant negative correlation between duration of sight recovery and visual cortical activity in the calcarine sulcus, suggesting that the longer the period of sight recovery, the lower the primary visual cortex activation during auditory processing. Importantly, although those individuals with the longest duration of visual recovery were also those with the oldest ages, the present results cannot be fully accounted for by an aging effect, because there was no relationship between age and visual cortex activation in the group of sighted controls. Alternatively, the negative correlation between activation in the calcarine sulcus and duration of sight recovery could represent an aging effect that is specific to the group of cataract-reversal individuals. However, because age and duration of sight recovery were highly correlated in the present sample ( $\tau=0.99, p<0.001)$, a more likely explanation for this correlation is that it represents the effect of sight recovery (Dormal et al., 2015) rather than an aging effect. Therefore, the present results corroborate the notion that crossmodal responses decrease over time (Rouger et al., 2012; Dormal et al., 2015) such that, after an extensive period of sight recovery, crossmodal plasticity during auditory processing is hardly observed. Specifically, crossmodal plasticity is only found in a correlational analysis (whereby the shorter the period of visual recovery, the higher the visual cortical responses to auditory stimulation; present study) and in multisensory conditions involving concurrent visual stimulation (Guerreiro et al., 2015). These results resemble those obtained in the domain of auditory restoration but after late-onset deafness: although crossmodal plasticity after cochlear implantation in deaf individuals is often reported (Doucet et al., 2006; Buckley and Tobey, 2011; Sandmann et al., 2012; Stropahl et al., 2015), a recent longitudinal study found that crossmodal visual responses within auditory areas decrease after auditory restoration, eventually reaching the level observed in normally hearing controls (Rouger et al., 2012).

In summary, the present study reveals that early visual deprivation affects the neural bases of auditory processing. This was 
observed as a lack of auditory cortex modulation by noise level in individuals who lacked developmental vision. That is, unlike normally sighted controls, who exhibited higher auditory cortex activation as noise level increased together with an increased recruitment of frontal and parietal areas involved in attentional control, cataract-reversal individuals displayed comparable auditory cortex activation across noise levels. Despite this lack of auditory cortex modulation, the behavioral performance of cataract-reversal individuals did not differ from that of normally sighted controls, suggesting that, under more demanding auditory conditions (e.g., low SNR), cataract-reversal individuals exhibit more efficient cortical processing of auditory stimuli (cf. Stevens and Weaver, 2009). Future studies should investigate the generalizability of the present findings to other auditory tasks involving nonspeech stimuli, as well as make use of other methodological techniques to measure neural synchronization across areas involved in auditory processing.

\section{References}

Bedny M, Pascual-Leone A, Dodell-Feder D, Fedorenko E, Saxe R (2011) Language processing in the occipital cortex of congenitally blind adults. Proc Natl Acad Sci U S A 108:4429-4434. CrossRef Medline

Bedny M, Pascual-Leone A, Dravida S, Saxe R (2012) A sensitive period for language in the visual cortex: distinct patterns of plasticity in congenitally versus late blind adults. Brain Lang 122:162-170. CrossRef Medline

Bedny M, Richardson H, Saxe R (2015) "Visual" cortex is active during language processing in young blind children. J Neurosci 35:11674-11681. CrossRef Medline

Bronchti G, Schönenberger N, Welker E, Van der Loos H (1992) Barrelfield expansion after neonatal eye removal in mice. Neuroreport 3:489-492. CrossRef Medline

Buckley KA, Tobey EA (2011) Cross-modal plasticity and speech perception in pre- and postlingually deaf cochlear implant users. Ear Hear 32:2-15. CrossRef Medline

Collignon O, Vandewalle G, Voss P, Albouy G, Charbonneau G, Lassonde M, Lepore F (2011) Functional specialization for auditory-spatial processing in the occipital cortex of congenitally blind humans. Proc Natl Acad Sci U S A 108:4435-4440. CrossRef Medline

Collignon O, Dormal G, Albouy G, Vandewalle G, Voss P, Phillips C, Lepore F (2013) Impact of blindness onset on the functional organization and the connectivity of the occipital cortex. Brain 136:2769-2783. CrossRef Medline

Crémieux J, Veraart C, Wanet-Defalque MC (1986) Effects of deprivation of vision and vibrissae on goal-directed locomotion in cats. Exp Brain Res 65:229-234. Medline

De Martino F, Moerel M, van de Moortele PF, Ugurbil K, Goebel R, Yacoub E, Formisano E (2013) Spatial organization of frequency preference and selectivity in the human inferior colliculus. Nat Commun 4:1386. CrossRef Medline

De Martino F, Moerel M, Xu J, van de Moortele PF, Ugurbil K, Goebel R, Yacoub E, Formisano E (2015) High-resolution mapping of myeloarchitecture in vivo: Localization of auditory areas in the human brain. Cereb Cortex 25:3394-3405. CrossRef Medline

Dietrich S, Hertrich I, Ackermann H (2013) Ultra-fast speech comprehension in blind subjects engages primary visual cortex, fusiform gyrus, and pulvinar-a functional magnetic resonance imaging (fMRI) study. BMC Neurosci 14:74. CrossRef Medline

Dormal G, Lepore F, Harissi-Dagher M, Albouy G, Bertone A, Rossion B, Collignon O (2015) Tracking the evolution of crossmodal plasticity and visual functions before and after sight restoration. J Neurophysiol 113: 1727-1742. CrossRef Medline

Doucet ME, Bergeron F, Lassonde M, Ferron P, Lepore F (2006) Crossmodal reorganization and speech perception in cochlear implant users. Brain 129:3376-3383. CrossRef Medline

Elbert T, Sterr A, Rockstroh B, Pantev C, Müller MM, Taub E (2002) Expansion of the tonotopic area in the auditory cortex of the blind. J Neurosci 22:9941-9944. Medline

Föcker J, Best A, Hölig C, Röder B (2012) The superiority in voice processing of the blind arises from neural plasticity at sensory processing stages. Neuropsychologia 50:2056-2067. CrossRef Medline
Forman SD, Cohen JD, Fitzgerald M, Eddy WF, Mintun MA, Noll DC (1995) Improved assessment of significant activation in functional magnetic resonance imaging (fMRI): use of a cluster-size threshold. Magn Reson Med 33:636-647. CrossRef Medline

Goebel R, Esposito F, Formisano E (2006) Analysis of functional image analysis content (FIAC) data with BrainVoyager QX: from single-subject to cortically aligned group general linear model analysis and selforganizing group independent component analysis. Hum Brain Mapp 27:392-401. CrossRef Medline

Gougoux F, Belin P, Voss P, Lepore F, Lassonde M, Zatorre RJ (2009) Voice perception in blind persons: a functional magnetic resonance imaging study. Neuropsychologia 47:2967-2974. CrossRef Medline

Guerreiro MJ, Putzar L, Röder B (2015) The effect of early visual deprivation on the neural bases of multisensory processing. Brain 138:1499 1504. CrossRef Medline

Hertrich I, Dietrich S, Moos A, Trouvain J, Ackermann H (2009) Enhanced speech perception capabilities in a blind listener are associated with activation of fusiform gyrus and primary visual cortex. Neurocase 15: 163-170. CrossRef Medline

Hölig C, Föcker J, Best A, Röder B, Büchel C (2014a) Brain systems mediating voice identity processing in blind humans. Hum Brain Mapp 35: 4607-4619. CrossRef Medline

Hölig C, Föcker J, Best A, Röder B, Büchel C (2014b) Crossmodal plasticity in the fusiform gyrus of late blind individuals during voice recognition. Neuroimage 103:374-382. CrossRef Medline

Huettel SA, Song AW, McCarthy G (2009) Functional magnetic resonance imaging, Ed 2. Sunderland, MA: Sinauer.

King AJ, Parsons CH (1999) Improved auditory spatial acuity in visually deprived ferrets. Eur J Neurosci 11:3945-3956. CrossRef Medline

Korte M, Rauschecker JP (1993) Auditory spatial tuning of cortical neurons is sharpened in cats with early blindness. J Neurophysiol 70:1717-1721. Medline

Kriegeskorte N, Simmons WK, Bellgowan PS, Baker CI (2009) Circular analysis in systems neuroscience: the dangers of double dipping. Nat Neurosci 12:535-540. CrossRef Medline

Kujala T, Alho K, Paavilainen P, Summala H, Nätänen R (1992) Neural plasticity in processing of sound location by the early blind: an eventrelated potential study. Electroencephalogr Clin Neurophysiol 84: 469-472. CrossRef Medline

Lewis TL, Maurer D (2005) Multiple sensitive periods in human visual development: evidence from visually deprived children. Dev Psychobiol 46: 163-183. CrossRef Medline

Lewkowicz DJ, Röder B (2012) Development of multisensory processing and the role of early experience. In: The new handbook of multisensory processes (Stein BE, ed), pp 607-626. Cambridge, MA: Massachusetts Institute of Technology.

Moerel M, De Martino F, Formisano E (2012) Processing of natural sounds in human auditory cortex: tonotopy, spectral tuning, and relation to voice sensitivity. J Neurosci 32:14205-14216. CrossRef Medline

Moerel M, De Martino F, Santoro R, Ugurbil K, Goebel R, Yacoub E, Formisano E (2013). Processing of natural sounds: characterization of multipeak spectral tuning in human auditory cortex. J Neurosci 33: 11888-11898. CrossRef Medline

Niemeyer W, Starlinger I (1981) Do the blind hear better? Investigations on auditory processing in congenital or early acquired blindness. II. Central functions. Audiology 20:510-515. CrossRef Medline

Owen AM, McMillan KM, Laird AR, Bullmore E (2005) N-back working memory paradigm: a meta-analysis of normative functional neuroimaging studies. Hum Brain Mapp 25:46-59. CrossRef Medline

Poldrack RA (2007) Region of interest analysis for fMRI. Soc Cogn Affect Neurosci 2:67-70. CrossRef Medline

Ptak R (2012) The frontoparietal attention network of the human brain: action, saliency, and a priority map of the environment. Neuroscientist 18:502-515. CrossRef Medline

Putzar L, Goerendt I, Lange K, Rösler F, Röder B (2007) Early visual deprivation impairs multisensory interactions in humans. Nat Neurosci 10: 1243-1245. CrossRef Medline

Putzar L, Hötting K, Röder B (2010) Early visual deprivation affects the development of face recognition and of audio-visual speech perception. Restor Neurol Neurosci 28:251-257. CrossRef Medline

Putzar L, Gondan M, Röder B (2012) Basic multisensory functions can be 
acquired after congenital visual pattern deprivation in humans. Dev Neuropsychol 37:697-711. CrossRef Medline

Rauschecker JP, Kniepert U (1994) Auditory localization behavior in visually deprived cats. Eur J Neurosci 6:149-160. CrossRef Medline

Rauschecker JP, Korte M (1993) Auditory compensation for early blindness in cat cerebral cortex. J Neurosci 13:4538-4548. Medline

Rauschecker JP, Tian B, Korte M, Egert U (1992) Crossmodal changes in the somatosensory vibrissa/barrel system of visually deprived animals. Proc Natl Acad Sci U S A 89:5063-5067. CrossRef Medline

Röder B, Rösler F, Hennighausen E, Näcker F (1996) Event-related potentials during auditory and somatosensory discrimination in sighted and blind human subjects. Brain Res Cogn Brain Res 4:77-93. CrossRef Medline

Röder B, Stock O, Bien S, Neville H, Rösler F (2002) Speech processing activates visual cortex in congenitally blind humans. Eur J Neurosci 16: 930-936. CrossRef Medline

Rouger J, Lagleyre S, Démonet JF, Fraysse B, Deguine O, Barone P (2012) Evolution of crossmodal reorganization of the voice area in cochlear-implanted deaf patients. Hum Brain Mapp 33:1929-1940. CrossRef Medline

Saenz M, Lewis LB, Huth AG, Fine I, Koch C (2008) Visual motion area $\mathrm{MT}+/ \mathrm{V} 5$ responds to auditory motion in human sight-recovery subjects. J Neurosci 28:5141-5148. CrossRef Medline

Sandmann P, Dillier N, Eichele T, Meyer M, Kegel A, Pascual-Marqui RD, Marcar VL, Jäncke L, Debener S (2012) Visual activation of auditory cortex reflects maladaptive plasticity in cochlear implant users. Brain 135: 555-568. CrossRef Medline
Starlinger I, Niemeyer W (1981) Do the blind hear better? Investigations on auditory processing in congenital or early acquired blindness I. Peripheral functions. Audiology 20:503-509. CrossRef Medline

Stevens AA, Weaver KE (2009) Functional characteristics of auditory cortex in the blind. Behav Brain Res 196:134-138. CrossRef Medline

Stilla R, Hanna R, Hu X, Mariola E, Deshpande G, Sathian K (2008) Neural processing underlying tactile microspatial discrimination in the blind: a functional magnetic resonance imaging study. J Vis 8:1-19. CrossRef Medline

Stropahl M, Plotz K, Schönfeld R, Lenarz T, Sandmann P, Yovel G, De Vos M, Debener S (2015) Cross-modal reorganization in cochlear implant users: auditory cortex contributes to visual face processing. Neuroimage 121:159-170. CrossRef Medline

Toldi J, Farkas T, Völgyi B (1994) Neonatal enucleation induces crossmodal changes in the barrel cortex of rat: a behavioral and electrophysiological study. Neurosci Lett 167:1-4. CrossRef Medline

Watkins KE, Shakespeare TJ, O’Donoghue MC, Alexander I, Ragge N, Cowey A, Bridge H (2013) Early auditory processing in area V5/MT+ of the congenitally blind brain. J Neurosci 33:18242-18246. CrossRef Medline

Wong PC, Uppunda AK, Parrish TB, Dhar S (2008) Cortical mechanisms of speech perception in noise. J Speech Lang Hear Res 51:1026-1041. CrossRef Medline

Wong PC, Jin JX, Gunasekera GM, Abel R, Lee ER, Dhar S (2009) Aging and cortical mechanisms of speech perception in noise. Neuropsychologia 47:693-703. CrossRef Medline 